\title{
An Intuitionistic Fuzzy Soft Set Theoretic Approach to Decision Making Problems
}

\author{
Surendra Singh Gautam, Abhishekh and S. R. Singh* \\ Department of Mathematics, Institute of Science \\ Banaras Hindu University, Varanasi-221005, India \\ *Corresponding author: srsingh@bhu.ac.in
}

Article history

Received: 16 November 2016

Received in revised form: 15 October 2017

Accepted: 15 February 2018

Published on line: 1 June 2018

\begin{abstract}
In general most of the real life problem of decision making involve imprecise parameters. In recent years, major emphasis of research workers in this area have been to develop the reliable models to deal with such imprecision and vagueness effectively. Several theories have been developed such as fuzzy set theory, interval valued fuzzy set, intuitionistic fuzzy set, and interval valued intuitionistic fuzzy set, rough set and soft set. The primary objectives of all the above developed theories are to deal with various kinds of uncertainty, imprecision and vagueness but unfortunately every theory has certain limitations. In the present paper we briefly introduced the notion of soft set, fuzzy soft set and intuitionistic fuzzy soft set. We extend the Jurio et al construction method of converting fuzzy set into intuitionistic fuzzy set to fuzzy soft set into intuitionistic fuzzy soft set. Here we consider a problem of decision making in fuzzy soft set and presented a method to generalize it into intuitionistic fuzzy soft set based decision making problem for modelling the problem in a better way. In the process we used the construction method and score function of intuitionistic fuzzy number.
\end{abstract}

Keywords Fuzzy soft set; intuitionistic fuzzy soft set; score function.

Mathematics Subject Classification 032E72, 90B50

\section{Introduction}

It is difficult to remove uncertainty and imprecision involved in the data frequently appearing in many real life problem. Among these, the decision making problem is one of the important field arising in the area of business, management, economics and many more area of science and technology. The uncertainty in data is traditionally dealt by probability theory but now a days many non-traditional theories such as fuzzy set theory introduced by Zadeh [1], Atanassov [2] presented a intuitionistic fuzzy set theory, Pawlak [3] presented a rough set theory and Gau and Buehrer [4] give the notion of vague set theory are being used to deal it comfortably. The concept of soft set given by Molodtsov [5]can be seen as new mathematical theory capable of dealing with uncertainty. Once the soft set theory was introduced, it was Maji et al. [6,7] who 
enriched the soft set theory by deriving its various properties. Maji et al. [8,9] further extended the soft set theory to fuzzy soft set and intuitionistic fuzzy soft set.

Roy and Maji [10] proposed the concept of a fuzzy soft set and studied its application in decision making under an imprecise environment. They attached a degree with the parameterization of fuzzy sets while defining a fuzzy soft set. Maji et al. [8] and Xu et al. [11] extended soft sets to intuitionistic fuzzy soft sets and vague soft sets respectively. Yang et al. [12] presented the concept of the interval-valued fuzzy soft sets by combining the interval-valued intuitionistic fuzzy set and soft set models. Kovkov et al. [13] considered the optimization problems in the framework of the theory of soft sets which is directed to formalization of the concept of approximate object description. Zau and Xiao [14] presented data analysis approaches of soft sets under incomplete information. It was Feng et al. [15] who gave a deep insights into decision making problem based on fuzzy soft sets. They discussedthe validity of the Roy-Maji method and showed its true limitations by means of level soft sets, and then presented an adjustable approach to fuzzy soft set based decision making. Further, Xiao et al. [16] introduced fuzzy parameterized (FP) soft set theory and Yang et al. [17] introduced the concept of multi-fuzzy soft set which is a combination of multi-fuzzy soft set and studied its basic operations.

This paper aims to present some results on an application of intuitionistic fuzzy soft sets in decision making problem. In addition some operation on an intuitionistic fuzzy soft set is defined such as union operation and intersection operation and also presents the construction method for converting the fuzzy soft set into intuitionistic fuzzy soft sets. The rest of this paper is organized as follows: section 2 introduces the basic definitions of soft sets, fuzzy soft sets and intuitionistic fuzzy soft set and the concept of intuitionistic fuzzy soft set with its operation and its construction method is presented. A decision making in intuitionistic fuzzy soft set and algorithm is presented in section 3 and application of intuitionistic fuzzy soft set in decision making problem discussed in section 4 and finally conclusion of the study is stated in section 5 .

\section{Preliminaries}

In this section we will briefly discuss the notions of soft sets, fuzzy soft sets and intuitionistic fuzzy soft sets, definition of score function and construction method of intuitionistic fuzzy number.

Definition 1 Let $U$ be an initial universe of objects and $E$ be the set of parameters in relation to objects in $U$. Consider a non empty set $A$ s.t. $A \subseteq E$. A pair $(f, A)$ is called a soft set over $U$, where $f$ is a mapping given by $f: A \rightarrow \wp(U)$, where $\wp(U)$ is power set of $U$.

Here $f$ is called approximate function of the soft set $(f, A)$ and the value $f(x)$ may be considered as the set of $\varepsilon$ - element of the soft set $(f, A)$ for $\varepsilon \in A$. Thus a soft set over $U$ can be represented by the set of ordered pairs $(f, A)=\{(\varepsilon, f(\varepsilon)): \varepsilon \in A, f(\varepsilon) \in \wp(U)$.

Example 1 Let $U=\left\{u_{1} u_{2}, \ldots, u_{7}\right.$ be a universal set and $E=\left\{h_{1} h_{2} h_{3} h_{4} h_{5}\right.$ be a set of parameters. If $A=\left\{h_{1} h_{2} h_{3} h_{4} \subseteq E, f\left(h_{1}\right)=\left\{u_{1} u_{3}, f\left(h_{2}\right)=\left\{u_{4}, f\left(h_{3}\right)=\left\{u_{1} u_{3} u_{5}, f\left(h_{4}\right)=U\right.\right.\right.\right.$ then the soft set $(f A)$ is written by $(f A)=\left\{\left(h_{1},\left\{u_{1}, u_{3}\right\}\right),\left(h_{2}\left\{u_{4}\right\}\right),\left(h_{3}\left\{u_{1}, u_{3}, u_{5}\right\}\right),\left(h_{4} U\right)\right\}$.

Definition 2 Let $\tilde{F}(U)$ be the set of all fuzzy subsets in universe $U$. Let $E$ be set of parameters and $A \subseteq E$. A pair $(\tilde{f}, A)$ is called a fuzzy soft set over $U$ where $\tilde{f}$ is mapping given by 
$\tilde{f}: A \rightarrow \tilde{F}(U)$.

Before introducing the notion of the intuitionistic fuzzy soft sets, Let us introduce the concept of intuitionistic fuzzy sets which is given by Atanassov $[2,18]$.

An intuitionistic fuzzy set $A$ defined over a universe of discourse $U$ is an object in the following form $A=\left\{\left\langle x \mu_{A}(x), \nu_{A}(x)>: x \in U\right\}\right.$, where the function $\mu_{A}: U \rightarrow[0,1]$ and $\nu_{A}: U \rightarrow[0,1]$ are the membership and non membership function respectively, of every element $x \in U$ to set $A$ and $0 \leq \mu_{A}(x)+\nu_{A}(x) \leq 1$ for every $x \in U$.

By introducing the concept of intuitionistic fuzzy sets into the theory of soft sets, Maji et al. $[9,19]$ proposed the concept of the intuitionistic fuzzy soft sets as follows.

Definition 3 Let $U$ be an initial universe of objects and $E$ be the set of parameters in relation to objects in $U$. Let $\tilde{F}^{I}(U)$ denote the set of all intuitionistic fuzzy sets of $U$ and $A \subseteq E$. A pair $\left(\tilde{f}^{I}, A\right)$ is an intuitionistic fuzzy soft set over $U$, where $\tilde{f}^{I}$ is a mapping given by $\tilde{f}^{I}: \bar{A} \rightarrow \tilde{F}^{I}(U)$.

For any parameter $\varepsilon \in A, \tilde{f}^{I}(\varepsilon)$ is an intuitionistic fuzzy subset of $U$ and it is called intuitionistic fuzzy value set of parameter $\varepsilon$. Clearly, $\tilde{f}^{I}(\varepsilon)$ can be written as an intuitionistic fuzzy set such that $\tilde{f}^{I}(\varepsilon)=\left\{\left\langle x, \mu_{\tilde{f}^{\prime}(\varepsilon)}(x), \nu_{\tilde{f}^{\prime}(\varepsilon)}(x): x \in U\right\}\right.$, where $\mu_{\tilde{f}^{I}(\varepsilon)}(x)$ and $\nu_{\tilde{f}^{I}(\varepsilon)}(x)$ be the membership and non-membership functions respectively. If $\forall x \in U, \gamma_{\tilde{f}^{I}(\varepsilon)}(x)=1-\mu_{\tilde{f}^{I}(\varepsilon)}(x)$ then $\tilde{f}^{I}(\varepsilon)$ will degenerated to be a traditional fuzzy soft set.

Definition 4 Let $\left(\tilde{f}^{I} A\right)$ and $\left(\tilde{g}^{I} B\right)$ be two intuitionistic fuzzy soft sets on the same universal set $U$. Then the union operation of $\left(\tilde{f}^{I} A\right)$ and $\left(\tilde{g}^{I} B\right)$ denoted by $\left(\tilde{f}^{I}, A\right) \tilde{\cup}\left(\tilde{g}^{I} B\right)$ is an intuitionistic fuzzy soft set defined as $\left(\tilde{f}^{I}, A\right) \tilde{\cup}\left(\tilde{g}^{I}, B\right)=\left(\tilde{h}^{I} C\right)$, where $C=A \cup B$ and $\forall \varepsilon \in C$

$$
h(\varepsilon)= \begin{cases}\tilde{f}^{I}(\varepsilon) & \varepsilon \in A-B, \\ \tilde{g}^{I}(\varepsilon) & \varepsilon \in B-A, \\ \tilde{f}^{I}(\varepsilon) \cup \tilde{g}^{I}(\varepsilon) & \varepsilon \in A \cup B .\end{cases}
$$

where $\left.\tilde{f}^{I}(\varepsilon) \cap \tilde{g}^{I}(\varepsilon)=\left\{\left\langle x, \max \left(\mu_{A}(x), \mu_{B}(x)\right), \min \left(\nu_{A}(x), \nu_{B}(x)\right)\right\rangle\right): x \in U\right\}$.

Definition 5 Let $\left(\tilde{f}^{I} A\right)$ and $\left(\tilde{g}^{I} B\right)$ be two intuitionistic fuzzy soft sets on the same universal set $U$. Then the intersection of $\left(\tilde{f}^{I} A\right)$ and $\left(\tilde{g}^{I} B\right)$ denoted by $\left(\tilde{f}^{I}, A\right) \tilde{\cap}\left(\tilde{g}^{I} B\right)$ is an intuitionistic fuzzy soft set defined as $\left(\tilde{f}^{I} A\right) \tilde{\cap}\left(\tilde{g}^{I} B\right)=\left(\tilde{h}^{I} C\right)$, where $C=A \cup B$ and $\forall \varepsilon \in C$

$$
h(\varepsilon)= \begin{cases}\tilde{f}^{I}(\varepsilon) & \varepsilon \in A-B, \\ \tilde{g}^{I}(\varepsilon) & \varepsilon \in B-A \\ \tilde{f}^{I}(\varepsilon) \cap \tilde{g}^{I}(\varepsilon) & \varepsilon \in A \cap B .\end{cases}
$$

where $\left.\tilde{f}^{I}(\varepsilon) \cap \tilde{g}^{I}(\varepsilon)=\left\{\left\langle x, \min \left(\mu_{A}(x), \mu_{B}(x)\right), \max \left(\nu_{A}(x), \nu_{B}(x)\right)\right\rangle\right): x \in U\right\}$.

Definition 6 (Xu and Yager [20]) Let $\alpha_{1}=\left\langle\mu_{\alpha_{1}}, \nu_{\alpha_{1}}\right\rangle$ and $\alpha_{2}=\left\langle\mu_{\alpha_{2}}, \nu_{\alpha_{2}}\right\rangle$ be two IFNs, $s\left(\alpha_{1}\right)=\mu_{\alpha_{1}}-\nu_{\alpha_{1}}$ and $s\left(\alpha_{2}\right)=\mu_{\alpha_{2}}-\nu_{\alpha_{2}}$ the scores of the IFNs $\alpha_{1}$ and $\alpha_{2}$ respectively, and $h\left(\alpha_{1}\right)=\mu_{\alpha_{1}}+\nu_{\alpha_{1}}$ and $h\left(\alpha_{2}\right)=\mu_{\alpha_{2}}+\nu_{\alpha_{2}}$ the accuracy degrees of the IFNs $\alpha_{1}$ and $\alpha_{2}$ respectively. Then

1. If $s\left(\alpha_{1}\right)<s\left(\alpha_{2}\right)$, then the IFN $\alpha_{1}$ is smaller than the IFN $\alpha_{2}$, denoted by $\alpha_{1}<\alpha_{2}$. 
2. If $s\left(\alpha_{1}\right)=s\left(\alpha_{2}\right)$, then

(a) If $h\left(\alpha_{1}\right)=h\left(\alpha_{2}\right)$, the IFNs $\alpha_{1}$ and $\alpha_{2}$ are equal, i.e. $\mu_{\alpha_{1}}=\mu_{\alpha_{2}}$ and $\nu_{\alpha_{1}}=\nu_{\alpha_{2}}$, denoted by $\alpha_{1}=\alpha_{2}$;

(b) If $h\left(\alpha_{1}\right)<h\left(\alpha_{2}\right)$, the IFN $\alpha_{1}$ is smaller than the IFN $\alpha_{2}$, denoted by $\alpha_{1}<\alpha_{2}$;

(c) If $h\left(\alpha_{1}\right)>h\left(\alpha_{2}\right)$, the IFN $\alpha_{1}$ is larger than $\alpha_{2}$, denoted by $\alpha_{1}>\alpha_{2}$.

\section{Construction Method of Intuitionistic Fuzzy Set}

A method of construction of Intuitionistic fuzzy set from fuzzy set given by Jurio et al. [21] is presented. In this method we represent the membership and non-membership degrees of each element.

Let $\mathrm{A}_{\mathrm{F}} \in \mathrm{FS}_{\mathrm{s}}(\mathrm{U})$ where $\mathrm{FS}_{\mathrm{s}}(\mathrm{U})$ denotes the set of all fuzzy sets in the Universal set $\mathrm{U}$ and let $\pi, \delta: \mathrm{U} \longrightarrow[0,1]$ be two mappings. Then

$$
\mathrm{I}=\left\{\left\langle\mu_{1}, f\left(\mu_{\mathrm{AF}}\left(\mu_{i}\right), \pi\left(\mu_{i}\right), \delta\left(\mu_{i}\right)\right)\right\rangle u_{i} \in \mathrm{U}\right\}
$$

is an Atanassov Intuitionistic fuzzy set(IFS) corresponding fuzzy set $A_{F}$, where the mapping

$$
f:[0,1]^{2} \times[0,1] \longrightarrow \mathrm{L}^{*}
$$

Given by $f(x, y, \delta)=\left(f_{\mu}(x, y, \delta), f_{\nu}(x, y, \delta)\right)$, where

$$
\begin{aligned}
& \left.\left.f_{\mu}(x, y, \delta)\right) x, y, \delta\right)=x(1-\delta y), \\
& f_{\nu}(x, y, \delta)=1-x(1-\delta y)-\delta y \\
& \mathrm{~L}^{*}=\{(x, y):(x, y) \in[0,1] \times[0,1] \text { and } x+y \leq 1\}
\end{aligned}
$$

satisfies that

1. If $y_{1} \leq y_{2}$ then $\pi\left(f\left(x, y_{1}, \delta\right)\right) \leq \pi\left(f\left(x, y_{2}, \delta\right)\right)$ for all $x, \delta \in[0,1]$

2. $f_{\mu}(x, y, \delta) \leq x \leq 1-f_{\nu}(x, y, \delta)$ for all $x \in[0,1]$

3. $f(x, 0, \delta)=(x, 1-x)$

4. $f(0, y, \delta)=(0,1-\delta y)$

5. $f(x, y, 0)=(x, 1-x)$

6. $\pi(f(x, y, \delta))=\delta y$

Example 2 Suppose

$$
\begin{aligned}
& \tilde{F}_{A}= \\
& \left\{\left(x_{1},\left\{\frac{u_{1}}{0.4}, \frac{u_{2}}{0.6}, \frac{u_{3}}{0.5}, \frac{u_{4}}{0.8}, \frac{u_{5}}{1}\right\}\right),\left(x_{2},\left\{\frac{u_{1}}{1}, \frac{u_{2}}{0.5}, \frac{u_{3}}{0.5}, \frac{u_{4}}{1}, \frac{u_{5}}{0.7}\right\}\right),\right. \\
& \left.\left(x_{3},\left\{\frac{u_{1}}{0.5}, \frac{u_{2}}{0.6}, \frac{u_{3}}{0.8}, \frac{u_{4}}{0.8}, \frac{u_{5}}{0.7}\right\}\right),\left(x_{4},\left\{\frac{0.4}{u_{1}}, \frac{0.7}{u_{21}}, \frac{0.6}{u_{3}}, \frac{0.9}{u_{4}}, \frac{1}{u_{5}}\right\}\right),\left(x_{5},\left\{\frac{u_{1}}{1}, \frac{u_{2}}{0.6}, \frac{u_{3}}{0.5}, \frac{u_{4}}{1}, \frac{u_{5}}{1}\right\}\right)\right\}
\end{aligned}
$$


is the fuzzy soft set. By using the above method fuzzy soft set converted in intuitionistic fuzzy soft set given as follows:

$$
\begin{aligned}
\tilde{F}_{A}^{\prime}= & \left\{\left(x_{1},\left\{\left\langle u_{1}, 0.24,0.36\right\rangle,\left\langle u_{2}, 0.36,0.24\right\rangle,\left\langle u_{3}, 0.3,0.3\right\rangle,\left\langle u_{4}, 0.48,0.12\right\rangle,\left\langle u_{5}, 0.6,0\right\rangle\right\}\right),\right. \\
& \left(x_{2},\left\{\left\langle u_{1}, 0.5,0\right\rangle,\left\langle u_{2}, 0.25,0.25\right\rangle,\left\langle u_{3}, 0.25,0.25\right\rangle,\left\langle u_{4}, 0.5,0\right\rangle,\left\langle u_{5}, 0.35,0.15\right\rangle\right\}\right), \\
& \left(x_{3},\left\{\left\langle u_{1}, 0.3,0.3\right\rangle,\left\langle u_{2}, 0.36,0.24\right\rangle,\left\langle u_{3}, 0.48,0.12\right\rangle,\left\langle u_{4}, 0.48,0.12\right\rangle,\left\langle u_{5}, 0.42,0.18>\right\}\right),\right. \\
& \left(x_{4},\left\{\left\langle u_{1}, 0.24,0.36\right\rangle,\left\langle u_{2}, 0.42,0.18\right\rangle,\left\langle u_{3}, 0.36,0.24\right\rangle,\left\langle u_{4}, 0.54,0.06\right\rangle,\left\langle u_{5}, 0.6,0\right\rangle\right\}\right), \\
& \left.\left(x_{5},\left\{\left\langle u_{1}, 0.5,0\right\rangle,\left\langle u_{2}, 0.3,0.2\right\rangle,\left\langle u_{3}, 0.25,0.25\right\rangle,\left\langle u_{4}, 0.5,0\right\rangle,\left\langle u_{5}, 0.4,0\right\rangle\right\}\right)\right\} .
\end{aligned}
$$

\section{Intuitionistic Fuzzy Soft Set in Decision Making}

In this section we present an intuitionistic fuzzy soft sets based decision making problem by extending the approach to fuzzy soft sets based decision making presented by Roy and Maji [10]. Let $\mathrm{U}=\left\{u_{1} u_{2}, \ldots u_{p}\right\}$ be a set of $p$-objects, which may be characterized by a set of parameters $\left\{A_{1} A_{2}, \ldots, A_{i}\right\}$. The parameter space $E$ may be written as $\left\{A_{1} \cup A_{2} \cup \ldots \cup A_{i} \subseteq E\right.$. Let each parameter set $A_{i}$ represent the $i^{\text {th }}$ class of parameter and the elements of $A_{i}$ represents a specific property set.

Comparison table is a square table in which columns both are labeled by the object names $u_{1}, u_{2}, \ldots, u_{n}$ of the universe and the entries $c_{i j}, i j=1,2, \ldots, n$ given by $c_{i j}=$ the number of parameters for which the intuitionistic fuzzy number of $u_{i}$ exceeds or equal to the intuitionistic fuzzy number of $u_{j}$.

Clearly $0 \leq c_{i j} \leq l$ and $c_{i j}=l, \forall i j$ where $l$ is the number of parameters present in a intuitionistic fuzzy soft set.

Thus $c_{i j}$ indicates a numerical measure, which is an integer number and $u_{i}$ dominates $u_{j}$ in $c_{i j}$ number of parameters out of $p$ parameters.

Let $r_{i}$ and $c_{i}$ be the row sum of an object $u_{i}$ and column sum of an object $u_{j}$ respectively can be calculated as $r_{i}=\sum_{j=1}^{n} c_{i j}$ and $c_{j}=\sum_{i=1}^{n} c_{i j}$. Clearly $r_{i}$ indicates the total number of parameters in which $u_{i}$ dominates all the member of $U$ and $c_{j}$ indicates the total number of parameters in which $u_{j}$ is dominated by all the members of $U$.

The Score of an object $u_{i}$ is $s_{i}$ may be given as $s_{i}=r_{i}-c_{j}$.

\section{Algorithm}

1. Identify the parameters and alternatives of the intuitionistic fuzzy soft sets.

2. Input the intuitionistic fuzzy soft set $\left(\tilde{f}^{I} A\right),\left(\tilde{g}^{I} B\right)$ and $\left(\tilde{h}^{I} C\right)$ with the appropriate parameter set $P$ as observed by the decision maker.

3. Compute the corresponding resultant intuitionistic fuzzy soft set $\left(\tilde{S}^{I} P\right)$ from the intuitionistic fuzzy soft sets $\left(\tilde{f}^{I} A\right),\left(\tilde{g}^{I} B\right)$ and $\left(\tilde{h}^{I} C\right)$ and place in tabular form.

4. Construct the comparison table of the intuitionistic fuzzy soft set $\left(\tilde{S}^{I} P\right)$ and compute $r_{i}$ and $c_{i}$ for $u_{i}, \forall i$.

5. Compute the Score of $u_{i}, \forall i$.

6. The optimal decision is to select $u_{k}$ if $S_{k}=s_{i}$. 
7. If $k$ has more than one value then any one of $u_{k}$ may be chosen.

\section{Numerical Illustration}

In this section we discussed the problem taken by Roy and Maji [10] in intuitionistic fuzzy soft set by using the method construction of intuitionistic fuzzy number from fuzzy number.

Let $U=\left\{u_{1}, u_{2}, u_{3}, u_{4}, u_{5}, u_{6}\right\}$ be the set of objects having different colors, sizes and surface texture features. The set of parameters is given by

$$
E=\left\{e_{1}, e_{2}, e_{3}, e_{4}, e_{5}, e_{6}, e_{7}, e_{8}, e_{9}, e_{10}, e_{11}, e_{12}, e_{13}\right\}
$$

where $e_{i}$ stand for blackish $\left(e_{1}\right)$, dark brown $\left(e_{2}\right)$, yellowish $\left(e_{3}\right)$, reddish $\left(e_{4}\right)$, large $\left(e_{5}\right)$, small $\left(e_{6}\right)$, very small $\left(e_{7}\right)$, average $\left(e_{8}\right)$, very large $\left(e_{9}\right)$, course $\left(e_{10}\right)$, moderately course $\left(e_{11}\right)$, fine $\left(e_{12}\right)$, extra fine $\left(e_{13}\right)$. Let $A=\left\{e_{1}, e_{2}, e_{3}, e_{4}\right\}, B=\left\{e_{5}, e_{6}, e_{7}, e_{8}, e_{9}\right\}$ and $C=\left\{e_{10}, e_{11}, e_{12}, e_{13}\right\}$ be three subsets of the set of parameter $E$. $A, B$ and $C$ represent the colour space, size of the object and surface texture granularity respectively.

Consider the intuitionistic fuzzy soft set $\left(\tilde{f}^{I} A\right),\left(\tilde{g}^{I} B\right)$ and $\left(\tilde{h}^{I} C\right)$ describe the objects having color space, object having size and texture features of the object surface. The problem is to identify an unknown object from the multi observes intuitionistic fuzzy data, specified by different decision makers in terms of intuitionistic fuzzy soft sets $\left(\tilde{f}^{I} A\right),\left(\tilde{g}^{I} B\right)$ and $\left(\tilde{h}^{I} C\right)$. The intuitionistic fuzzy soft sets may be computed as follows. The intuitionistic fuzzy soft $\operatorname{set}\left(\tilde{f}^{I} A\right)$ is defined as

$$
\begin{aligned}
&\left(\tilde{f}^{I}, A\right)=\left(e_{1}=\left\{\left\langle u_{1}, 0.22,0.51\right\rangle,\left\langle u_{2}, 0.22,0.51\right\rangle,\left\langle u_{3}, 0.29,0.44\right\rangle,\left\langle u_{4}, 0.58,0.15\right\rangle,\right.\right. \\
&\left.\left\langle u_{5}, 0.51,0.22\right\rangle,\left\langle u_{6}, 0.66,0.07\right\rangle\right\}, e_{2}=\left\{\left\langle u_{1}, 0.33,0.49\right\rangle,\left\langle u_{2}, 0.74,0.08\right\rangle,\left\langle u_{3}, 0.41,0.41\right\rangle,\right. \\
&\left.\left\langle u_{4}, 0.16,0.66\right\rangle,\left\langle u_{5}, 0.25,0.57\right\rangle,\left\langle u_{6}, 0.16,0.66\right\rangle\right\}, e_{3}=\left\{\left\langle u_{1}, 0.46,0.30\right\rangle,\left\langle u_{2}, 0.23,0.53\right\rangle,\right. \\
&\left.\left\langle u_{3}, 0.61,0.15\right\rangle,\left\langle u_{4}, 0.30,0.46\right\rangle,\left\langle u_{5}, 0.46,0.30\right\rangle,\left\langle u_{6}, 0.30,0.46\right\rangle\right\}, e_{4}=\left\{\left\langle u_{1}, 0.66,0.07\right\rangle,\right. \\
&\left.\left.\left\langle u_{2}, 0.365,0.365\right\rangle,\left\langle u_{3}, 0.51,0.22\right\rangle,\left\langle u_{4}, 0.58,0.15\right\rangle,\left\langle u_{5}, 0.365,0.365\right\rangle,\left\langle u_{6}, 0.22,0.51\right\rangle\right\}\right) \\
&\left(\tilde{g}^{I}, B\right)=\left(e_{5}=\left\{\left\langle u_{1}, 0.33,0.49\right\rangle,\left\langle u_{2}, 0.66,0.16\right\rangle,\left\langle u_{3}, 0.49,0.33\right\rangle,\left\langle u_{4}, 0.74,0.08\right\rangle\right.\right. \\
&\left.\left\langle u_{5}, 0.16,0.66\right\rangle,\left\langle u_{6}, 0.25,0.57\right\rangle\right\}, \\
& e_{6}=\left\{\left\langle u_{1}, 0.18,0.74\right\rangle,\left\langle u_{2}, 0.55,0.37\right\rangle,\left\langle u_{3}, 0.37,0.55\right\rangle,\right. \\
&\left.\left\langle u_{4}, 0.74,0.18\right\rangle,\left\langle u_{5}, 0.09,0.83\right\rangle,\left\langle u_{6}, 0.18,0.75\right\rangle\right\}, e_{7}=\left\{\left\langle u_{1}, 0.66,0.16\right\rangle,\left\langle u_{2}, 0.25,0.57\right\rangle,\right. \\
&\left.\left\langle u_{3}, 0.33,0.49\right\rangle,\left\langle u_{4}, 0.16,0.66\right\rangle,\left\langle u_{5}, 0.74,0.08\right\rangle,\left\langle u_{6}, 0.66,0.16\right\rangle\right\}, e_{8}=\left\{\left\langle u_{1}, 0.55,0.37\right\rangle,\right. \\
&\left.\left\langle u_{2}, 0.09,0.83\right\rangle,\left\langle u_{3}, 0.09,0.83\right\rangle,\left\langle u_{4}, 0.09,0.83\right\rangle,\left\langle u_{5}, 0.74,0.18\right\rangle,\left\langle u_{6}, 0.55,0.37\right\rangle\right\} \\
& e_{9}=\left\{\left\langle u_{1}, 0.325,0.325\right\rangle,\left\langle u_{2}, 0.45,0.19\right\rangle,\left\langle u_{3}, 0.455,0.195\right\rangle,\left\langle u_{4}, 0.39,0.26\right\rangle,\right. \\
&\left.\left.\left\langle u_{5}, 0.455,0.195\right\rangle,\left\langle u_{6}, 0.325,0.325\right\rangle\right\}\right) . \\
&\left(\tilde{h}^{I}, C\right)=\left(e_{10}=\left\{\left\langle u_{1}, 0.22,0.51\right\rangle,\left\langle u_{2}, 0.44,0.29\right\rangle,\left\langle u_{3}, 0.365,0.365\right\rangle,\left\langle u_{4}, 0.51,0.22\right\rangle,\right.\right. \\
&\left.\left\langle u_{5}, 0.44,0.29\right\rangle,\left\langle u_{6}, 0.66,0.07\right\rangle\right\}, e_{1} 1=\left\{\left\langle u_{1}, 0.29,0.43\right\rangle,\left\langle u_{2}, 0.36,0.36\right\rangle,\left\langle u_{3}, 0.43,0.29\right\rangle,\right. \\
&\left.\left\langle u_{4}, 0.43,0.29\right\rangle,\left\langle u_{5}, 0.43,0.29\right\rangle,\left\langle u_{6}, 0.50,0.22\right\rangle\right\}, e_{1} 2=\left\{\left\langle u_{1}, 0.09,0.84\right\rangle,\left\langle u_{2}, 0.37,0.56\right\rangle,\right. \\
&\left.\left\langle u_{3}, 0.28,0.65\right\rangle,\left\langle u_{4}, 0.56,0.37\right\rangle,\left\langle u_{5}, 0.465,0.465\right\rangle,\left\langle u_{6}, 0.65,0.28\right\rangle\right\}, e_{1} 3=\left\{\left\langle u_{1}, 0.66,0.07\right\rangle,\right. \\
&\left.\left.\left\langle u_{2}, 0.365,0.365\right\rangle,\left\langle u_{3}, 0.44,0.29\right\rangle,\left\langle u_{4}, 0.22,0.51\right\rangle,\left\langle u_{5}, 0.29,0.44\right\rangle,\left\langle u_{6}, 0.66,0.07\right\rangle\right\}\right) .
\end{aligned}
$$


The tabular representation of the intuitionistic fuzzy soft $\operatorname{sets}\left(\tilde{f}^{I} A\right),\left(\tilde{g}^{I} B\right)$ and $\left(\tilde{h}^{I} C\right)$ are shown in Table 1, Table 2 and Table 3 respectively.

Table 1: Tabular Representation of the Intuitionistic Fuzzy Soft Set $\left(\tilde{f}^{I} B\right)$

\begin{tabular}{|c|c|c|c|c|}
\hline$U$ & $\operatorname{Blackish}\left(e_{1}\right)$ & $\operatorname{Dark} \operatorname{brown}\left(e_{2}\right)$ & Yellowish $\left(e_{3}\right)$ & $\operatorname{Reddish}\left(e_{4}\right)$ \\
\hline$u_{1}$ & $\langle 0.22,0.51\rangle$ & $\langle 0.33,0.49\rangle$ & $\langle 0.46,0.30\rangle$ & $\langle 0.66,0.07\rangle$ \\
\hline$u_{2}$ & $\langle 0.22,0.51\rangle$ & $\langle 0.74,0.08\rangle$ & $\langle 0.23,0.53\rangle$ & $\langle 0.365,0.365\rangle$ \\
\hline$u_{3}$ & $\langle 0.29,0.44\rangle$ & $\langle 0.29,0.44\rangle$ & $\langle 0.61,0.15\rangle$ & $\langle 0.51,0.22\rangle$ \\
\hline$u_{4}$ & $\langle 0.58,0.15\rangle$ & $\langle 0.16,0.66\rangle$ & $\langle 0.30,0.46\rangle$ & $\langle 0.58,0.15\rangle$ \\
\hline$u_{5}$ & $\langle 0.51,0.22\rangle$ & $\langle 0.25,0.57\rangle$ & $\langle 0.46,0.30\rangle$ & $\langle 0.365,0.365\rangle$ \\
\hline$u_{6}$ & $\langle 0.66,0.07\rangle$ & $\langle 0.16,0.66\rangle$ & $\langle 0.30,0.46\rangle$ & $\langle 0.22,0.51\rangle$ \\
\hline
\end{tabular}

Table 2: Tabular representation of the intuitionistic fuzzy soft set $\left(\tilde{g}^{I} B\right)$

\begin{tabular}{|c|c|c|c|c|c|}
\hline$U$ & Large $\left(e_{5}\right)$ & Very large $\left(e_{6}\right)$ & $\operatorname{Small}\left(e_{7}\right)$ & Very Small $\left(e_{8}\right)$ & Average $\left(e_{9}\right)$ \\
\hline$u_{1}$ & $\langle 0.33,0.49\rangle$ & $\langle 0.18,0.74\rangle$ & $\langle 0.66,0.16\rangle$ & $\langle 0.55,0.37\rangle$ & $\langle 0.325,0.325\rangle$ \\
\hline$u_{2}$ & $\langle 0.66,0.16\rangle$ & $\langle 0.55,0.37\rangle$ & $\langle 0.25,0.57\rangle$ & $\langle 0.09,0.83\rangle$ & $\langle 0.455,0.195\rangle$ \\
\hline$u_{3}$ & $\langle 0.49,0.33\rangle$ & $\langle 0.37,0.55\rangle$ & $\langle 0.25,0.57\rangle$ & $\langle 0.09,0.83\rangle$ & $\langle 0.455,0.195\rangle$ \\
\hline$u_{4}$ & $\langle 0.74,0.08\rangle$ & $\langle 0.74,0.18\rangle$ & $\langle 0.16,0.66\rangle$ & $\langle 0.09,0.83\rangle$ & $\langle 0.39,0.26\rangle$ \\
\hline$u_{5}$ & $\langle 0.16,0.66\rangle$ & $\langle 0.09,0.83\rangle$ & $\langle 0.74,0.08\rangle$ & $\langle 0.74,0.18\rangle$ & $\langle 0.455,0.195\rangle$ \\
\hline$u_{6}$ & $\langle 0.25,0.57\rangle$ & $\langle 0.18,0.75\rangle$ & $\langle 0.66,0.16\rangle$ & $\langle 0.55,0.37\rangle$ & $\langle 0.325,0.325\rangle$ \\
\hline
\end{tabular}

Let $\left(\tilde{f}^{I} A\right)$ and $\left(\tilde{g}^{I} B\right)$ be any two intuitionistic fuzzy soft sets over the universe $U$. Taking the union of above two intuitionistic fuzzy soft set of $\left(\tilde{f}^{I} A\right)$ and $\left(\tilde{g}^{I} B\right)$ we get the another intuitionistic fuzzy soft set. The obtained intuitionistic fuzzy soft set is termed as resultant intuitionistic fuzzy soft set of $\left(\tilde{f}^{I}, A\right)$ and $\left(\tilde{g}^{I} B\right)$.

The resultant intuitionistic fuzzy soft set of $\left(\tilde{f}^{I} A\right)$ and $\left(\tilde{g}^{I} B\right)$ have $4 \times 5=20$ parameters of the form $a_{i j}$, where $a_{i j}=e_{i} \vee e_{j}$ for all $i=1,2,3,4$ and $j=1,2,3,4,5$. If we require the intuitionistic fuzzy soft set for the parameter $R=\left\{a_{11}, a_{15}, a_{21}, a_{24}, a_{33}, a_{44}, a_{45}\right\}$. For the intuitionistic fuzzy soft set $\left(\tilde{f}^{I} A\right)$ and $\left(\tilde{g}^{I}, B\right)$ the resultant intuitionistic fuzzy soft set $\left(\tilde{k}^{I} R\right)$ (say) are shown in Table 4.

Consider the intuitionistic fuzzy soft sets $\left(\tilde{f}^{I} A\right),\left(\tilde{g}^{I} B\right)$ and $\left(\tilde{h}^{I} C\right)$. Let

$$
P=\left\{a_{11} \vee e_{10} a_{15} \vee e_{12} a_{21} \vee e_{11} a_{24} \vee e_{13} a_{33} \vee e_{12} a_{44} \vee e_{12} a_{45} \vee e_{13}\right\}
$$

be the set of choice parameters of an observer. Then the resultant intuitionistic fuzzy soft set with parameters $P$ is $\left(\tilde{S}^{I} P\right)$ placed in Table 5 . The comparison table of the above resultant intuitionistic fuzzy soft set is shown in Table 6. We compute the row-sum, column-sum and the score for each $u_{i}$ as shown in Table 7 .

From the above score table it is clear that $u_{6}$ has maximum score, So decision is in favour of selecting $u_{6}$. 
Table 3: Tabular Representation of the Intuitionistic Fuzzy Soft Set $\left(\tilde{h}^{I} C\right)$

\begin{tabular}{|c|c|c|c|c|}
\hline$U$ & Course $\left(e_{10}\right)$ & Moderately course $\left(e_{11}\right)$ & Fine $\left(e_{12}\right)$ & Extra Fine $\left(e_{13}\right)$ \\
\hline$u_{1}$ & $\langle 0.22,0.51\rangle$ & $\langle 0.29,0.43\rangle$ & $\langle 0.09,0.84\rangle$ & $\langle 0.66,0.07\rangle$ \\
\hline$u_{2}$ & $\langle 0.44,0.29\rangle$ & $\langle 0.36,0.36\rangle$ & $\langle 0.37,0.56\rangle$ & $\langle 0.365,0.365\rangle$ \\
\hline$u_{3}$ & $\langle 0.365,0.365\rangle$ & $\langle 0.43,0.29\rangle$ & $\langle 0.28,0.65\rangle$ & $\langle 0.44,0.29\rangle$ \\
\hline$u_{4}$ & $\langle 0.51,0.22\rangle$ & $\langle 0.43,0.29\rangle$ & $\langle 0.56,0.37\rangle$ & $\langle 0.22,0.51\rangle$ \\
\hline$u_{5}$ & $\langle 0.44,0.29\rangle$ & $\langle 0.43,0.29\rangle$ & $\langle 0.465,0.465\rangle$ & $\langle 0.29,0.44\rangle$ \\
\hline$u_{6}$ & $\langle 0.66,0.07\rangle$ & $\langle 0.50,0.22\rangle$ & $\langle 0.65,0.28\rangle$ & $\langle 0.66,0.07\rangle$ \\
\hline
\end{tabular}

Table 4: Tabular Representation of the Resultant Intuitionistic Fuzzy Soft Set $\left(\tilde{k}^{I}, R\right)$

\begin{tabular}{|c|c|c|c|c|c|c|c|}
\hline$U$ & $a_{11}$ & $a_{15}$ & $a_{21}$ & $a_{24}$ & $a_{33}$ & $a_{44}$ & $a_{45}$ \\
\hline$u_{1}$ & $\langle 0.33,0.49\rangle$ & $\langle 0.325,0.325\rangle$ & $\langle 0.33,0.49\rangle$ & $\langle 0.55,0.37\rangle$ & $\langle 0.66,0.16\rangle$ & $\langle 0.66,0.07\rangle$ & $\langle 0.66,0.07\rangle$ \\
\hline$u_{2}$ & $\langle 0.66,0.16\rangle$ & $\langle 0.22,0.51\rangle$ & $\langle 0.74,0.08\rangle$ & $\langle 0.74,0.08\rangle$ & $\langle 0.25,0.53\rangle$ & $\langle 0.365,0.365\rangle$ & $\langle 0.445,0.195\rangle$ \\
\hline$u_{3}$ & $\langle 0.49,0.33\rangle$ & $\langle 0.455,0.195\rangle$ & $\langle 0.49,0.33\rangle$ & $\langle 0.455,0.195\rangle$ & $\langle 0.61,0.15\rangle$ & $\langle 0.51,0.22\rangle$ & $\langle 0.51,0.19\rangle$ \\
\hline$u_{4}$ & $\langle 0.74,0.08\rangle$ & $\langle 0.58,0.15\rangle$ & $\langle 0.74,0.08\rangle$ & $\langle 0.16,0.66\rangle$ & $\langle 0.30,0.46\rangle$ & $\langle 0.58,0.15\rangle$ & $\langle 0.58,0.15\rangle$ \\
\hline$u_{5}$ & $\langle 0.51,0.22\rangle$ & $\langle 0.51,0.195\rangle$ & $\langle 0.25,0.57\rangle$ & $\langle 0.74,0.18\rangle$ & $\langle 0.74,0.08\rangle$ & $\langle 0.74,0.18\rangle$ & $\langle 0.44,0.19\rangle$ \\
\hline$u_{6}$ & $\langle 0.66,0.07\rangle$ & $\langle 0.66,0.07\rangle$ & $\langle 0.25,0.57\rangle$ & $\langle 0.55,0.37\rangle$ & $\langle 0.66,0.16\rangle$ & $\langle 0.55,0.37\rangle$ & $\langle 0.325,0.325\rangle$ \\
\hline
\end{tabular}

Table 5: Tabular Representation of the Resultant Intuitionistic Fuzzy Soft Set $\left(\tilde{S}^{I} P\right)$

\begin{tabular}{|c|c|c|c|c|c|c|c|}
\hline & $a_{11} \vee e_{10}$ & $a_{15} \vee e_{12}$ & $a_{21} \vee e_{11}$ & $a_{24} \vee e_{13}$ & $a_{33} \vee e_{12}$ & $a_{44} v e e e_{12}$ & $a_{45} \vee e_{13}$ \\
\hline$u_{1}$ & $\langle 0.33,0.49\rangle$ & $\langle 0.325,0.325\rangle$ & $\langle 0.33,0.43\rangle$ & $\langle 0.66,0.07\rangle$ & $\langle 0.66,0.16\rangle$ & $\langle 0.66,0.07\rangle$ & $\langle 0.66,0.07\rangle$ \\
\hline$u_{2}$ & $\langle 0.66,0.16\rangle$ & $\langle 0.37,0.51\rangle$ & $\langle 0.74,0.08\rangle$ & $\langle 0.74,0.08\rangle$ & $\langle 0.37,0.53\rangle$ & $\langle 0.37,0.365\rangle$ & $\langle 0.455,0.195\rangle$ \\
\hline$u_{3}$ & $\langle 0.49,0.33\rangle$ & $\langle 0.455,0.195\rangle$ & $\langle 0.49,0.29\rangle$ & $\langle 0.455,0.195\rangle$ & $\langle 0.61,0.15\rangle$ & $\langle 0.51,0.22\rangle$ & $\langle 0.51,0.22\rangle$ \\
\hline$u_{4}$ & $\langle 0.74,0.08\rangle$ & $\langle 0.58,0.15\rangle$ & $\langle 0.74,0.08\rangle$ & $\langle 0.22,0.51\rangle$ & $\langle 0.56,0.37\rangle$ & $\langle 0.58,0.15\rangle$ & $\langle 0.58,0.15\rangle$ \\
\hline$u_{5}$ & $\langle 0.51,0.22\rangle$ & $\langle 0.51,0.195\rangle$ & $\langle 0.43,0.29\rangle$ & $\langle 0.43,0.29\rangle$ & $\langle 0.74,0.18\rangle$ & $\langle 0.74,0.18\rangle$ & $\langle 0.445,0.195\rangle$ \\
\hline$u_{6}$ & $\langle 0.66,0.07\rangle$ & $\langle 0.66,0.07\rangle$ & $\langle 0.50,0.22\rangle$ & $\langle 0.66,0.07\rangle$ & $\langle 0.66,0.16\rangle$ & $\langle 0.65,0.28\rangle$ & $\langle 0.66,0.07\rangle$ \\
\hline
\end{tabular}


Table 6: Comparison Table of the Intuitionistic Fuzzy Soft Set $\left(\tilde{S}^{I} P\right)$

\begin{tabular}{|l|l|l|l|l|l|l|}
\hline & $u_{1}$ & $u_{2}$ & $u_{3}$ & $u_{4}$ & $u_{5}$ & $u_{6}$ \\
\hline$u_{1}$ & 7 & 4 & 4 & 4 & 3 & 4 \\
\hline$u_{2}$ & 3 & 7 & 3 & 2 & 4 & 2 \\
\hline$u_{3}$ & 3 & 4 & 7 & 2 & 2 & 0 \\
\hline$u_{4}$ & 3 & 6 & 5 & 7 & 4 & 3 \\
\hline$u_{5}$ & 4 & 4 & 5 & 3 & 7 & 2 \\
\hline$u_{6}$ & 6 & 5 & 7 & 4 & 5 & 7 \\
\hline
\end{tabular}

Table 7: Score Table of the Intuitionistic Fuzzy Soft Set $\left(\tilde{S}^{I}, P\right)$

\begin{tabular}{|c|c|c|c|}
\hline & Row-sum $\left(r_{i}\right)$ & Column-sum $\left(c_{j}\right)$ & Score $\left(s_{i}\right)$ \\
\hline$u_{1}$ & 26 & 26 & 0 \\
\hline$u_{2}$ & 21 & 30 & -9 \\
\hline$u_{3}$ & 18 & 31 & -13 \\
\hline$u_{4}$ & 28 & 22 & 6 \\
\hline$u_{5}$ & 25 & 25 & 0 \\
\hline$u_{6}$ & 34 & 18 & 16 \\
\hline
\end{tabular}

\section{Conclusion}

In this paper, we consider a problem of decision making in fuzzy soft set theory and presented a method to generalize it into intuitionistic fuzzy soft set based decision making problem for modelling the problem in a better way. In the process we used the construction method and score function of intuitionistic fuzzy number. Here, our presented intuitionistic fuzzy soft set based decision making approach provide better result comparison to Roy and Maji [10].

\section{References}

[1] Zadeh, L. A. Fuzzy sets. Inform. Control. 1965. 8: 338-353.

[2] Atanassov, K. Intuitionistic fuzzy sets. Fuzzy Sets and Systems. 1986. 20: 87-96.

[3] Pawlak, Z. Rough sets. Int. J. of Inform. Comput. Sci. 1982. 11: 341-356.

[4] Gau, W. L. and Buehrer, D. J. Vague sets. IEEE Trans. System Man Cybernet. 1993. 23(2): $610-614$.

[5] Molodtsov, D. A. Soft set theory-first results. Comput. Math. Appl. 1999. 37: 19-31. 
[6] Maji, P. K., Biswas, R. and Roy, A. R. Fuzzy soft sets. Journal of Fuzzy Mathematics. 2001. 9(3): 589-602.

[7] Maji, P. Biswas, R. and Roy, A. R. Soft set theory. Comput, Math. Appl. 2003. 45(4-5): 555-562.

[8] Maji, P. K., Biswas, R. and Roy, A. R. Intuitionistic fuzzy soft sets. J. Fuzzy Math. 2001. 9(3): $677-692$.

[9] Maji, P. K., Roy, A. R. and Biswas, R. On intuitionistic fuzzy soft sets. J. Fuzzy Math. 2004. 12(3): 669-683.

[10] Roy, A. R. and Maji, P. K. A fuzzy soft set theoretic approach to decision making problems. Journal of Computational and Applied Mathematics. 2007. 203(2): 412-418.

[11] Xu, W., Ma, J., Wang, S. and Hao, G. Vague soft sets and their properties. Comput. Math. Appl. 2010. 59(2): 787-794.

[12] Yang, X. B., Lin, T. Y., Yang, J. Y., Li, Y. and Yu, D. Combination of interval-valued fuzzy set and soft set. Comput. Math. Appl. 2009. 58(3): 521-527.

[13] Kovkov, D. V., Kolbanov, V. M. and D. A. Molodtsov, D. A. Soft sets theory-based optimization. J. Comput. Sys. Sc. Int. 2007. 46(6): 872-880.

[14] Zou, Y. and Xiao, Z. Data analysis approaches of soft sets under incomplete information. Knowl. Base. Syst. 2008. 21: 941-945.

[15] Feng, F., Jun, Y. B., Liu, X. and Li, L. An adjustable approach to fuzzy soft set based decision making. Journal of Computational and Applied Mathematics. 2010. 234: 10-20.

[16] Xiao, Z., Gong, K. and Zou, Y. A Combined Forecasting Approach Based on Fuzzy Soft Sets. Journal of Computational and Applied Mathematics. 2010. 228: 326-333.

[17] Yang, Y., Tan, X. and Meng, C. The Multi-Fuzzy Soft Set and Its Application in Decision Making. Applied Mathematical Modelling. 2012. 37: 4915-4923.

[18] Atanassov, K. Intuitionistic Fuzzy Sets. Physica-verlag Heidelberg, New York. 1999.

[19] Maji, P. K.(2009). More on intuitionistic fuzzy soft sets, in: Proceedings of the 12th International Conference on Rough Sets, Fuzzy Sets, Data Mining and Granular Computing. In H. Sakai, M.K. Chakraborty. A.E. Hassanien, D. Slezak, W. Zhu (Eds.). Lecture Notes in Computer Science, 5908. Springer. RSFDGrC 2009. 231-240.

[20] Xu, Z., Yager, R. Some Geometric Aggregation Operators Based on Intuitionistic Fuzzy Sets. Int. J. of General Systems. 2006. 35(4): 417-433

[21] Jurio A, Paternain D, Bustince H, Guerra C, Beliakov G. A construction method of Atanassov's intuitionistic fuzzy sets for image processing. In Proceedings of the fifth IEEE conference on intelligent systems. 2010. 337-342. 